\title{
Diacronie
}

Studi di Storia Contemporanea

$N^{\circ} 27,3$ | 2016

Stato, costituzione e democrazia

\section{Alessandro Naccarato, Difendere la democrazia. Il PCI contro la lotta armata}

Federica Addis

\section{(2) OpenEdition \\ 1 Journals}

Edizione digitale

URL: http://journals.openedition.org/diacronie/4206

DOI: $10.4000 /$ diacronie.4206

ISSN: 2038-0925

Editore

Association culturelle Diacronie

Notizia bibliografica digitale

Federica Addis, «Alessandro Naccarato, Difendere la democrazia. II PCl contro la lotta armata », Diacronie [Online], $N^{\circ}$ 27, 3 | 2016, documento 19, Messo online il 29 septembre 2016, consultato il 23

septembre 2020. URL : http://journals.openedition.org/diacronie/4206 ; DOI : https://doi.org/10.4000/ diacronie.4206 


\section{Diacronie}

\section{RECENSIONE:}

\section{Alessandro NACCARATO, Difendere la democrazia. Il PCI contro la lotta armata, Roma, Carocci, 2015, 352 pp.}

a cura di Federica ADDIS *

Il lavoro di Alessandro Naccarato ${ }^{1}$ ha l'indubbio merito di indagare, per la prima volta in un'opera interamente dedicata, il rapporto tra il Partito Comunista Italiano e la lotta armata di sinistra degli anni Settanta ed Ottanta. Se eccettuiamo i contributi di Ermanno Taviani, significativi ma brevi e costretti in volumi collettanei di più ampio respiro², l'atteggiamento, l'interpretazione e la reazione del PCI al terrorismo "rosso" sono stati, per lungo tempo e sorprendentemente, poco presenti nell'agenda degli storici.

La narrazione di Naccarato si sviluppa lungo una linea cronologica che restituisce al lettore l'evoluzione delle posizioni assunte dal PCI verso il fenomeno terroristico, fornendo un preciso rendiconto di un largo spettro di contributi di diversi esponenti del Partito e di una parte del mondo intellettuale che ad esso si riferiva. Sono, infatti, raccolti o riportati alla memoria le pubblicazioni su «l'Unità» e «Rinascita» e i discorsi pubblici, le riunioni del Comitato Centrale e quelle della Sezione Problemi dello Stato, così come gli interventi in sede parlamentare e il conseguente contributo legislativo per la difesa della democrazia. Se da una parte uno degli indubitabili pregi del volume è quello di fondarsi su una base documentaria solida, varia ed in molti casi inedita, dall'altra la linea della restituzione letterale e cronologica dei documenti indagati,

\footnotetext{
${ }^{1}$ Alessandro Naccarato è professore di Lettere e Storia e deputato del Partito Democratico. Sul tema ha già pubblicato: Violenze, eversione e terrorismo del partito armato a Padova, Padova, CLEUP, 2008.

2 TAVIANI, Ermanno, Il terrorismo rosso, la violenza e la crisi della cultura politica del PCI, in VENTRONE, Angelo (a cura di), I dannati della rivoluzione. Violenza politica e storia d'Italia negli anni Sessanta e Settanta, Macerata, EUM, 2010, pp. 129-154; TAVIANI, Ermanno, PCI, estremismo di sinistra e terrorismo, in DE ROSA Gabriele, MONINA Giancarlo, L'Italia repubblicana nella crisi degli anni settanta. Sistema politico e istituzioni, vol. IV, Soveria Mannelli, Rubbettino, 2003, pp. 235-275.
} 
seguita talvolta pedissequamente, lascia forse troppo poco spazio ad un'analisi interpretativa.

Il volume prende avvio dagli anni della contestazione studentesca. Emerge qui un Partito Comunista spiazzato dal movimento sessantottino, erroneamente convinto che si trattasse di un fenomeno di dimensioni ridotte e dal carattere transitorio. Il PCI si trovò sospeso tra la difesa delle istituzioni ed il costante tentativo di non rimanere emarginato dal movimento, da una parte per reintegrare frange di giovani nella politica istituzionale, dall'altra perché vedeva in quei contestatori un potenziale bacino elettorale. È in questa fase che l'interpretazione della violenza politica assunse quella forma entro cui il PCI rimase costretto per tutta la prima metà degli anni Settanta, la convinzione cioè di un estremismo creato od utilizzato in chiave anticomunista. Veniva ripresa ed attualizzata l'idea gramsciana di un «sovversivismo dall'alto», della protesta come strumento delle classi dominanti per determinare il bilanciamento del potere, di posizioni estremiste che avevano «oggettivamente contribuito al disegno moderato e reazionario di spostare a destra e mobilitare genericamente contro la sinistra l'opinione pubblica»3. Questa convinzione si affermò in tutta la sua portata nel 1972, con la morte di Giangiacomo Feltrinelli e del Commissario Luigi Calabresi. Se per la morte dell'editore Enrico Berlinguer parlò di «pesante sospetto di una spaventosa messa in scena» e di «torbidi disegni delle centrali di provocazione italiane e straniere»4, l'omicidio Calabresi venne inserito, per via di un'analisi fondata sul criterio del cui prodest, in una più ampia "trama nera" iniziata con Piazza Fontana. Fu in questi termini che venne letta la virulenta apparizione delle Brigate Rosse nel 1974. Il sequestro Sossi divenne così uno strumento per influenzare il referendum sul divorzio, mentre il gruppo armato fu messo all'indice come banda criminale o pseudorivoluzionaria, concretamente anticomunista e reazionaria e parte integrante della strategia della tensione.

Cominciava a delinearsi come necessario uno studio più approfondito dei gruppi estremisti, ma soprattutto una mobilitazione popolare e di massa capace, sotto coordinamento dal partito, di isolare le frange estremiste ed evitare ulteriori provocazioni. Per questa via fu organizzato, nel gennaio del 1975, il primo seminario nazionale sull'estremismo alla scuola delle Frattocchie e fu istituita, l'anno successivo,

3 COSSUTTA, Armando, «Sovversivismo delle classi dominanti ed estremismo", in Critica marxista, 10, 1/1972; NACCARATO, Alessandro, Difendere la democrazia. Il PCI contro la lotta armata, Roma, Carocci, 2015.

${ }^{4}$ "Le conclusioni di Berlinguer al XIII Congresso», in l'Unità, 18 marzo 1972; NACCARATO, Alessandro, Difendere la democrazia, cit., p. 55. 
la Sezione problemi dello Stato, una divisione della Direzioni dedita allo studio dei problemi relativi a sicurezza e giustizia, in particolare in relazione al pericolo terrorista.

Se il 1977, nello specifico il famoso comizio di Lama alla Sapienza interrotto da gruppi di Autonomia Operaia, viene comunemente considerato il momento di rottura definitivo tra la sinistra istituzionale e i gruppi extra-parlamentari5, quell'anno detiene una funzione periodizzante anche per quanto concerne l'interpretazione del fenomeno eversivo da parte del Partito. A partire dal 1977, infatti, si cominciò a suggerire un'autocritica per aver sottovalutato il fenomeno eversivo o, peggio, per aver evitato posizioni eccessivamente severe nella speranza di ottenere consensi tra i giovani contestatori, mentre si cominciò a far luce sulla matrice di sinistra dell'eversione e sul suo legame, per quanto indiretto, con il Sessantotto. Fu Ugo Pecchioli, responsabile della Sezione problemi dello Stato, il primo a parlare di un "terrorismo di sinistra", per la cui analisi la comparazione con l'estremismo neofascista e l'idea di un sostegno statale od internazionale erano fuorvianti e poco utili. Rimase però costante la volontà di mantenere le distanze dall'eversione di sinistra, considerandola non solo estranea alle tradizioni di lotta del Partito Comunista Italiano, ma anche lontana dalla cultura e dalla morale del movimento operaio. Prova di questo atteggiamento fu la compattezza e le convinzione con la quale il Partito rigettò l'idea di un «album di famiglia» all'interno del quale ritrovare sia i brigatisti che il PCI degli anni Cinquanta, proposta da Rossana Rossanda su «il Manifesto» del 28 marzo 1978. Proprio al 1978 e al ruolo del Partito come garante della democrazia con il posizionamento sulla "linea della fermezza" durante il sequestro Moro, l'economia del volume lascia troppo poco spazio, affidandosi ad un rimando in nota che appare eccessivamente liquidatorio. Il baricentro dell'intero lavoro è d'altronde spostato nella fase successiva, dal 1979 in avanti. Complice il costante riferimento a Padova, città di provenienza dell'autore, Naccarato dedica ampio spazio alle vicende di Autonomia Operaia, che vedeva proprio nel Veneto e in particolare nell'ateneo patavino la propria roccaforte.

Sebbene l'approfondimento su Padova sia una costante già dai primi capitoli, esso assume primaria importanza a partire dai famosi arresti del 7 aprile 1979, con i quali fu interrotta l'attività dei vertici di Autonomia, in molti casi docenti dell'Università patavina. Il PCI assunse fin da subito una posizione di convinto supporto all'istruttoria del Procuratore di Padova Calogero, arrivando a scontrarsi con altre forze politiche (ad esempio il PSI) e con diversi intellettuali comunisti, critici verso un procedimento giudiziario ritenuto persecutorio, al limite del reato d'opinione e repressivo, e

5 Si veda ad esempio la discussione del volume di Naccarato del 12 gennaio 2016 a Palazzo Montecitorio, URL: < http://webtv.camera.it/archivio?id=8809\&position=0 > [consultato il 16 maggio 2016]. 
sostenitori di un generale "garantismo" nei confronti degli imputati. Il Partito non solo sostenne la correttezza formale del procedimento giudiziario, ma fu uno dei principali fautori dello schema interpretativo alla sua base. Proprio l'appoggio a quello che sarà noto come "Teorema Calogero", nel suo identificare oltre che un generale contatto tra organizzazioni extra-parlamentari e gruppi terroristici, un vero e proprio ruolo dirigenziale di Autonomia nei confronti delle Brigate Rosse, riportò definitivamente l’interpretazione del PCI entro i confini italiani, allontanandola in modo risolutorio dall'idea di manovre di "grandi vecchi" o di complotti internazionali in chiave anticomunista. Rimase però la convinzione che si trattasse di forze antidemocratiche ed antipopolari, per la cui sconfitta sarebbe stata necessaria una reazione ferma e compatta, soprattutto all'interno delle fabbriche. Le vicende di Autonomia consolidarono quindi il sempre più convinto appoggio del PCI agli organi dello stato, alla polizia e alla magistratura: fu così che il Partito promosse l'adozione della Legge n. 625 del 1979 concernente misure urgenti per la tutela dell'ordine democratico e della sicurezza pubblica, oltre che le leggi su pentitismo e dissociazione. Il volume si chiude con la vicenda di "Negri deputato": la sua elezione nelle liste del Partito Radicale portò la necessità di discutere in sede parlamentare dell'autorizzazione ai processi ed all'arresto nei suoi confronti. Fu l'occasione per un serrato confronto interno al PCI, in cui oscillazioni e posizioni differenti derivarono dalla delicatezza di dover decidere per l'arresto di un imputato già sottoposto ad una lunga carcerazione preventiva, e che si risolse nell'autorizzazione a procedere in giudizio, ma nell'astensione al voto per l'autorizzazione all'arresto ${ }^{6}$.

Il libro si distingue per aver saputo dare un rendiconto molto ampio, ma al contempo agile e preciso, dell'intervento teorico e pratico del PCI nei confronti della violenza politica di sinistra degli anni Settanta ed Ottanta, aggiungendo un tassello fondamentale alla ricerca storica sul tema. Il tratto peculiare dell'opera sta nell'accompagnare la narrazione principale ad un focus, costante e ricco di spunti, sulla dimensione locale. L'attenzione a Padova e all'esperienza autonoma toglie certo spazio ai primi anni Settanta, agli albori del fenomeno terroristico, una fase in cui il tentativo di non perdere alcune frange del movimento, legato ad una strategia diretta alla difesa delle istituzioni, lasciò un margine di ambiguità nella politica del PCI, tanto da far parlare addirittura di un atteggiamento di "doppiezza” del Partito verso la violenza politica. Concentrandosi prevalentemente sul "dopo-Moro" il volume tratteggia invece,

\footnotetext{
${ }^{6}$ La proposta comunista di sospendere la decisione sull'arresto fino alla sentenza della Corte d'Assise di Roma, che si sarebbe avuta di lì a breve, fu rigettata con voto alla Camera (decisiva per la mancata approvazione fu l'astensione dei Radicali).
} 
fin dal titolo, l'immagine di un Partito Comunista dedito in modo integro, fermo e compatto alla difesa della democrazia, il cui contributo fu fondamentale per il superamento dell'emergenza e la sconfitta dell'eversione nel rispetto dei limiti imposti dall'ordinamento democratico. Rimangono in questo senso da approfondire in maniera più pregnante alcune questioni già proposte da Ermanno Taviani in relazione, appunto, alla prima fase dell'eversione di sinistra, tra cui ad esempio le aporie della cultura politica di un Partito Comunista «che aveva rinnegato la violenza nella sua strategia (nella via italiana al socialismo) ma non nei suoi riferimenti ideologici di fondo»7, oltre che la presenza di radici ideologiche comuni all'estremismo e di rapporti concreti tra singole personalità e a livello di organizzazioni territoriali (almeno fino al 1975) ${ }^{8}$.

L’interesse per il padovano ha però alcuni vantaggi non trascurabili. Le vicende venete costringono innanzitutto l'autore ad analizzare in modo approfondito il rapporto tra fenomeno terroristico ed Autonomia Operaia, rilevando punti di contatto e aderenze teoriche poco contestabili. In questo senso il lavoro di Naccarato va nella stessa direzione di alcuni recenti studi, che, prediligendo un approccio di tipo storicoculturale e storico-sociale, mirano a rendere conto del contesto nel quale l'esperienza terroristica prese forma, dei contatti tra sinistra radicale ed esperienze di violenza, delle «radici culturali e politiche dell'estremismo di sinistra [...] spostando l'attenzione sui linguaggi della politica, sulle reti (incorporee) di significato e le reti (corporee) della militanza»9, ridimensionando l'interpretazione che ha visto nella violenza politica un «détournement rispetto ad un percorso radicale, ma democratico, di conflittualità e contestazione per la trasformazione politica ${ }^{10}$ e che ha così tratteggiato una linea di separazione tra la protesta positiva degli anni Sessanta e la degenerazione cupa e violenta del decennio successivo ${ }^{11}$.

Il volume mostra poi la fecondità di un approccio che esplora la dimensione locale dell'eversione di sinistra, facendone emergere segni specifici ed elementi caratterizzati, utili per una "mappatura" del fenomeno nella Penisola. La dimensione locale rappresenta d'altronde una strada ancora poco battuta dagli studi sul terrorismo, la quale, tuttavia, potrebbe contribuire a fornire elementi nuovi per lo studio di questo fenomeno anche su scala nazionale prestando attenzione ai diversi sviluppi nelle singole aree regionali.

\footnotetext{
7 TAVIANI, Ermanno, Il terrorismo rosso, cit., p. 104.

8 Ibidem, p. 103.

9 ARMANI, Barbara, «Italia anni settanta. Movimenti, violenza politica e lotta armata tra memoria e rappresentazione storiografica», in Storica, XI, 32, 2005, pp. 41-82, p. 63.

10 BETTA, Emmanuel (a cura di), «Violenza politica e anni Settanta. Questioni e itinerari di ricerca», in Contemporanea, 16, 4/2013, pp. 613-616, cit., p. 614.

${ }^{11}$ ARMANI, Barbara, «Italia anni Settanta», cit., pp. 43-51.
} 


\section{* L'autore}

Federica Addis è laureata in Scienze Politiche presso l'Università di Padova. È dottoranda borsista in Storia dell'Europa dal Medioevo all'Età Contemporanea presso l'Università degli Studi di Teramo. Il suo progetto di ricerca dottorale mira ad indagare l'atteggiamento della Repubblica Democratica Tedesca nei confronti della sinistra extra-parlamentare, della violenza politica e del fenomeno terroristico in Italia tra il 1968 e la fine degli anni Ottanta.

URL: < http://www.studistorici.com/progett/autori/\#Addis >

\section{Per citare questo articolo:}

ADDIS, Federica, «Recensione: Alessandro NACCARATO, Difendere la democrazia. II PCl contro la lotta armata, Roma, Carocci, 2015, 352 pp.», Diacronie. Studi di Storia Contemporanea : Stato, costituzione e democrazia, 29/09/2016,

URL:<http://www.studistorici.com/2016/09/29/addis_numero_27/ >

Diacronie Studi di Storia Contemporanea 0 www.diacronie.it

Risorsa digitale indipendente a carattere storiografico. Uscita trimestrale.

redazione.diacronie@hotmail.it

Comitato di redazione: Jacopo Bassi - Luca Bufarale - Elisa Grandi - Antonio César Moreno Cantano - Deborah Paci - Fausto Pietrancosta - Alessandro Salvador - Matteo Tomasoni - Luca Zuccolo

Diritti: gli articoli di Diacronie. Studi di Storia Contemporanea sono pubblicati sotto licenza Creative Commons 3.0. Possono essere riprodotti e modificati a patto di indicare eventuali modifiche dei contenuti, di riconoscere la paternità dell'opera e di condividerla allo stesso modo. La citazione di estratti è comunque sempre autorizzata, nei limiti previsti dalla legge. 